University for Business and Technology in Kosovo

UBT Knowledge Center

UBT International Conference

2012 UBT International Conference

Nov 2nd, 9:00 AM - Nov 3rd, 5:00 PM

\title{
Monetary Valuation of Voluntary Contributions: the Case of Lower Austria
}

Réka Heim

Danube University, reka.heim@donau-uni.ac.at

Andrea Hoeltl

Danube University, andrea.hoeltl@donau-uni.ac.at

Follow this and additional works at: https://knowledgecenter.ubt-uni.net/conference

Part of the Business Commons

\section{Recommended Citation}

Heim, Réka and Hoeltl, Andrea, "Monetary Valuation of Voluntary Contributions: the Case of Lower Austria" (2012). UBT International Conference. 35.

https://knowledgecenter.ubt-uni.net/conference/2012/all-events/35

This Event is brought to you for free and open access by the Publication and Journals at UBT Knowledge Center. It has been accepted for inclusion in UBT International Conference by an authorized administrator of UBT Knowledge Center. For more information, please contact knowledge.center@ubt-uni.net. 


\title{
Monetary Valuation of Voluntary Contributions: the Case of Lower Austria
}

\author{
Réka Heim ${ }^{1}$ and Andrea Hoelt $1^{2}$ \\ ${ }^{1,2}$ Center for Economic Research, Department for Management and Economics, Danube University Krems, Austria \\ reka.heim@donau-uni.ac.at; andrea.hoeltl@donau-uni.ac.at
}

\begin{abstract}
The circumstances of voluntary engagement have largely remained unexamined due to the vague and challenging description of their content. Voluntary engagement tends to increase in European nations characterized by civil societies with material prosperity and high life satisfaction such as in Norway, Sweden and in Austria.

This paper studies voluntary contributions in Lower Austria by tracking a cumulative data file from STATISTICS AUSTRIA in terms which purpose is twofold: first, in order to estimate the quantitative dimension of voluntary contributions, data from an additional section of the Austrian Micro Census is considered, and second employment and unemployment data from the section of the Austrian Micro Census that covers the European Labor Force Survey, to provide monetary valuation of voluntary contributions. Complementary insights into voluntary engagement in Lower Austria are delivered by expert interviews. Our results provide evidence of the extensive personal satisfactory importance of voluntary contributions and show disapproval towards monetary compensation of volunteering efforts.

Our identifying assumption is that $43.8 \%$ of all Austrians above 15 years of age are contributing voluntarily, which allows us to control for corresponding values for Lower Austria. We find that 617,804 persons in Lower Austria - which amounts to 46.8\% of the population - are voluntarily engaged in ten areas which are segmented into formal and into informal fields of voluntary involvement with neighborhood support taking top-tier with 28.6\% of all fields, where primarily females are present. Our analysis suggests that the monetary value of voluntary contributions in Lower Austria can be determined with $€ 3.3 \mathrm{bn}$.
\end{abstract}

Keywords: public economics, labor and demographic economics, labor force composition, wage level and structure, regional data

\section{Introduction}

Voluntary engagement accounts for partial empirical literature documentation with subject to public funding. Yet, as we argue, it is often difficult to separate voluntary engagement from ordinary employment categorization due to the vague and challenging description of its content. To the best of our knowledge, this is the first paper to consider monetary valuation of voluntary contributions in Austria in a detailed manner. Our paper contributes to a larger research agenda on the labor market transmis sion due to voluntary engagements.

In the European picture, civic engagement can be captured in Scandinavian countries, due to early educational sensitivities and to the absence of funding constraints which are prevalent in the remaining Euro area. The implication is that the stability of volunteerism in Norway and in Sweden with a participation quota of about 50\% points to a fundamental role of individuals as vehicles of civic engagement characterized by continuous economic growth and prosperity (Wollebæk et al., 2012). But the citizens' volunteering behavior shows large differences within the European Union. The "European Social Reality" survey (Eurobarometer, 2007) determines Austria with 60\%, the Netherlands with 55\% and Sweden with 53\% as the countries with a high level of volunteering. Fewer volunteers can be found in Lithuania (11\%) and Portugal (12\%). On average 35\% of the European citizens (EU25) volunteer. However, the number of volunteers has risen in Europe in the last ten years. According to Angermann and Sittermann (2010) there are prejudices with regard to voluntary work particularly in the countries of Central and Eastern Europe, because volunteering is strongly equated with obligatory activities in former socialistic countries. At the beginning of the 1990s many organizations had to be newly founded in these countries.

Additionally to the national programs, there are numerous programs on volunteering at the EU-level. Volunteering is considered as a possibility to promote life-long learning and as an important contribution to the promotion of active citizenship. For instance, the program „Europe for citizens“ (1904/2006/EC) facilitates transnational experiences for citizens. „Youth in Action“ is an EU program (1719/2006/EC) for the funding of (out-of-school) activities for young people in Europe. The program for Life-long Learning (LLP) is divided into various sub-programs that are based on the life and learning situation in question.The Austrian Council for Volunteering (2004) defines voluntary engagement as being organizational, associative or institutional framework bound with the duration and the regularity of its scope displaying a monotone devolution. Importantly, the primary characteristic of voluntary contributions towards societal progress is the third party component that is, excluding any perso nal or household internal efforts in order to foster non-monetary exchange in form of formal and of informal voluntary involvement. 
The remainder of this paper is organized as follows: Chapter 2 lays out the nature and the form of voluntary contributions with an economic perspective by introducing the key figures resulting from the segmentation of voluntary engagement into ten fields and by correspondingly adjusting the participation quotas of voluntary engagement in Lower Austria. Chapter 3 provides complementary evidence on the monetary valuation of voluntary contributions by using wage-tax data at the individual level to document gender-specific allocation of monetary compensations of voluntary efforts. Chapter 4 takes a broader qualitative approach to characterize the societal impact of voluntary contributions which have remained largely underestimated. Chapter 5 provides concluding remarks.

\section{Voluntary Contributions in an Economic Context}

This chapter presents evidence of the economic position and its relevance with respect to voluntary contributions. We argue that the volume of voluntary engagement provides predictions about the monetary valuation thereof, and in any case, these inquirie s do not suggest that individuals do consider the volume of voluntary engagement as having a negative relation on the societal values with respect to the consideration of monetary compensation of voluntary efforts.

Our results demonstrate that $43.8 \%^{109}$ (3.02 million persons in absolute terms) of all Austrians above 15 years of age are contributing voluntarily, which is below the $46.8 \%$ of all residents of Lower Austria who are above 15 years old and significantly above the $34.5 \%$ of all Viennese residents who are engaged in voluntary activities. The prevalence of most socio-demographic characteristics shows differences by age, by educational attainment and by marital status.

\subsection{Segmentation of Voluntary Engagement}

Since the pers pective of the monetary valuation of voluntary contributions seems manifold not only at a first glance, voluntary engagement has been segmented into nine formal and into one informal field of voluntary involvement, in order to adequately monitor the timely efforts made by individuals. The nine formal fields of voluntary engagements comprise of the following areas: "Emergency relief and rescue service"; "Arts, culture, entertainment and leisure time"; "Environment, nature and animal welfare"; "Churchly or religious sector"; "Social and health sector"; "Policy work and representation of interests"; "Civic and local community activities"; "Education" and "Sports and exercise". The informal field of voluntary engagement is represented by "Neighborhood support and informal sector".

We find that in Lower Austria 32.7\% of the citizens above 15 years of age are involved in a formal field (in organizations / in associations) whereas $28.6 \%$ contribute to informal ("neighborhood support") voluntary engagements. In terms of segmenting into gender relevant fields of voluntary engagement, more men are formally (with 37.6\%) than informally (with $27.7 \%$ ) active, while more females (with 29.5\%) are informally supportive compared to formal contributions of females (with $28.0 \%$ ). These results show deviations in terms of country-specific numbers which focus on the formal segment of voluntary engagement. In particular, $27.9 \%$ of all Austrian respondents are formally active, where men are taking top-tier with $33.0 \%$ and where women occupy the second rank with $23.2 \%$. In the informal area of mainly neighborhood support $27.1 \%$ of all Austrian respondents are engaged, allowing drawing strong similarities to Lower Austria at a first glance, but in terms of gender characteristics disp laying deviations, since $27.3 \%$ of women and $26.9 \%$ of men commit to informal voluntary activities in Austria.

Figure 1 below depicts the participation quotas in relative terms in all ten fields of voluntary engagement focusing on Lower Austria partially obtained from the Austrian Micro Census $4^{\text {th }}$ quarter 2006 (STATISTICS AUSTRIA, 2006). These results are therefore weighted and are subject to extrapolation concerning the number of respondents. The highest participation quota of $28.6 \%$ is attributed to the informal field of voluntary engagement. A segmentation of the formal fields of voluntary engagemen $\mathrm{t}$ relates to the highest participation quota of $9.0 \%$ in the "Churchly or religious sector", to the second rank with $7.8 \%$ attributed to the "Emergency relief and rescue service" area and to the third rank with $7.7 \%$ assigned to the formal field of "Arts, culture, entertainment and leisure time". The two lowest participation quotas of $2.7 \%$ and of $3.0 \%$ are represented by the areas of "Education" and of "Environment, nature and animal welfare", respectively.

Figure 1: Participation quotas in fields of voluntary engagement in percent

\footnotetext{
${ }^{109}$ The Albanian word 'peme' means 'tree' in English'. The last word 'acid' implies 'death'
} 


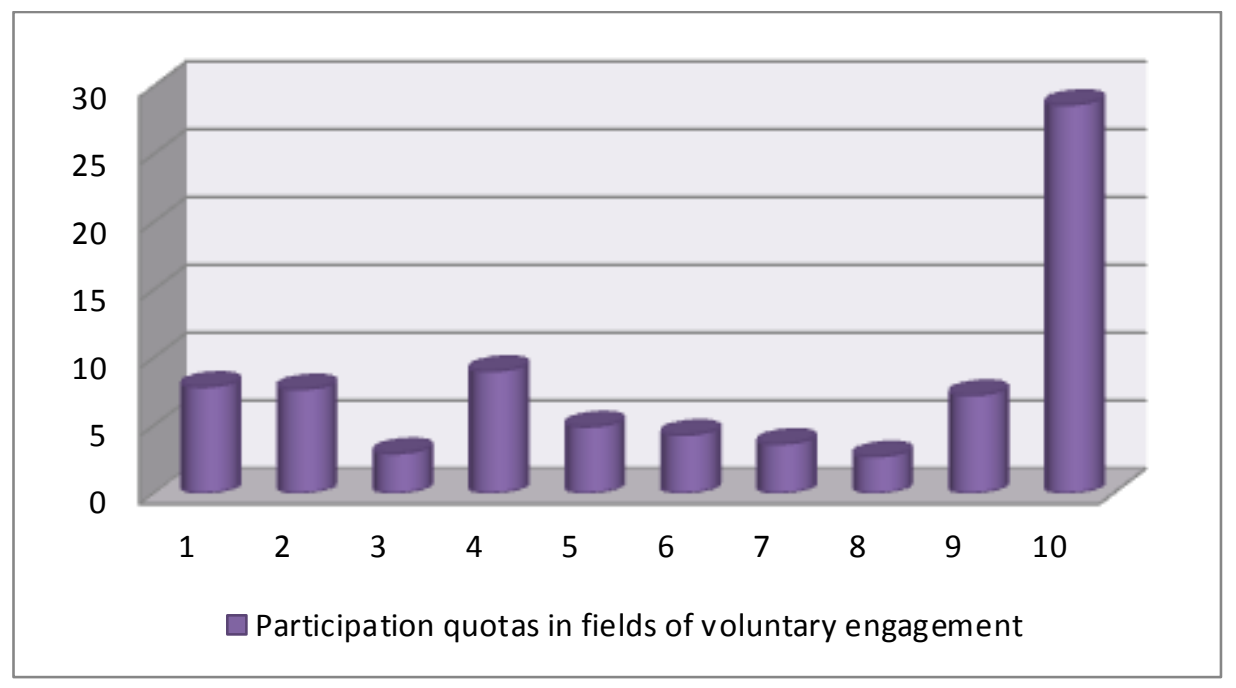

Source: STATISTICS AUSTRIA Austrian Micro Census $4^{\text {th }}$ quarter 2006 and authors’ own calculations.

Horizontal notations: 1=Emergency relief and rescue service; 2=Arts, culture, entertainment and leisure time; 3=Environment, nature and animal welfare; 4=Churchly or religious sector; 5=Social and health sector; 6=Policy work and representation of interests; 7=Civic and local community activities; 8=Education; 9=Sports and exercise; 10=Neighborhood support and informal sector

\subsection{Sociodemographic Characteristics of Voluntary Engagement}

This section is concerned with triple sociodemographic attributes that can be assigned to voluntary contributions, namely age, educational attainment and marital status in order to account for any dynamic characteristics stemming from demographic variables. By taking participation quotas in relationship to age of volunteers into account, we find that the first rank is occupied by the group of 30 to 49 -year olds with a quota of $42.3 \%$, followed by the group of 50 to 69 -year olds with a quota of $32.4 \%$ and with the group of 15 to 29-year olds occupying third rank with a participation quota of $17.4 \%$. The final rank represents persons above 70 years of age with a respectable participation quota of $7.9 \%$.

As far as the highest completed educational attainment is concerned, we find a strong impact of the obtained education degree on voluntary contributions. Around 36.5\% of Lower Austrians who graduated from a vocational higher education school are voluntarily engaged at the highest rank, followed by individuals with an obtained degree from a compulsory school with a participation quota of $18.7 \%$. The third highest rank is represented by persons who have graduated from a vocational middle school with a quota of $16.9 \%$. Interestingly, the lowest overall rank is attributed to individuals graduating from vocational higher education courses, such as colleges, with a participation quota of $13.7 \%$.

Our paper also uncovers additional insights about the participation quotas of volunteers with respect to their civil status. First, voluntary engagement is most pronounced for married individuals with an overwhelming participation quota of $63.9 \%$ followed by single persons with $24.4 \%$. The weakest group in terms of civil status that is voluntarily engaged is the grou p of divorced persons with a low participation rate of $5.7 \%$.

\subsection{The Volume of Voluntary Engagement}

In this paper we further analyze the volume of voluntary engagement in Lower Austria with an emphasis on the actual working hours on a weekly basis by initially defining the actual hours worked as the actual hours delivered per week. Further, we wish to note the introduction of the constraint referring to the inclusion of overtime but to the deduction of absenteeism such as le ave or sick leave.

As a consequence, we estimate the volume of voluntary engagement as 3,351,347 hours per week in total, where the timely efforts in the field of formal voluntary involvement (in organizations / in associations) amount to 1,900,644 hours per week and in the field of informal voluntary engagement ("neighborhood support") add up to 1,450,703 hours per week. In order to accurately monitor the volume of voluntary engagement on the individual level, we set the average of the actual hours worked per week and find that an individual is engaged 5.4 actual hours per week on average in formal and in informal duties. Correspondingly, the timely efforts in the field of formal voluntary involvement (in organizations / in associations) amount to an average of 4.1 actual hours per week and in the field of informal voluntary engagement ("neighborhood support") add up to an average of 3.8 actual hours per week on individual levels.

Figure 2 below displays the actual participation rates per week in hours in all ten fields of voluntary engagement with a segmentation towards gender. Importantly, we show that in the leading "Emergency relief and rescue service" area women on average are involved 4.9 hours per week, whereas men engage for 3.6 hours per week. Two further results are striking: first, in the field of "Neighborhood support and informal sector" women on average deliver 4.4 hours per week, whereas males are involved for 2.7 hours per week and second, in the formal field of "Arts, culture, entertainment and leisure time" men are engaged double of the average hours of women with 4.4 hours per week (males) compared to 2.2 hours per week (females). 
Figure 2: Gender-specific participation rates/week in hours in fields of voluntary engagement

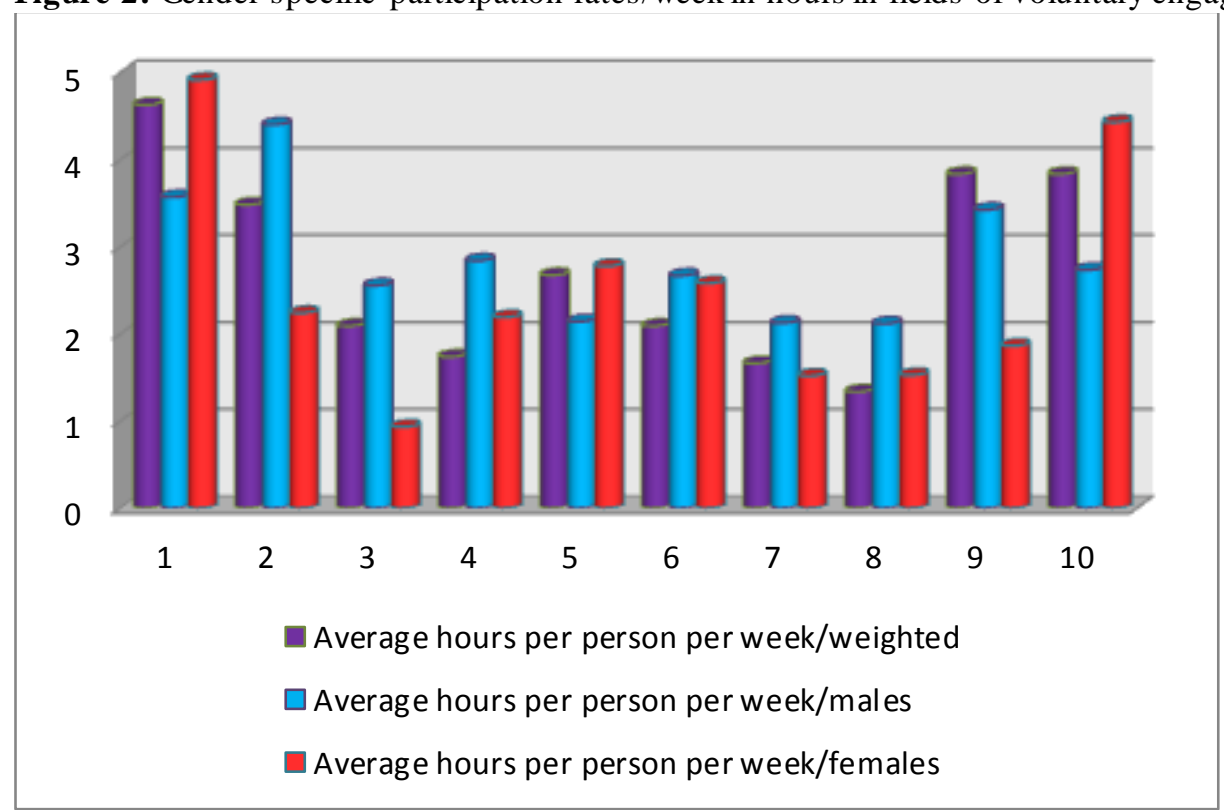

Source: STATISTICS AUSTRIA Austrian Micro Census $4^{\text {th }}$ quarter 2006 and authors'own calculations.

Horizontal notations: 1=Emergency relief and rescue service; $2=$ Arts, culture, entertainment and leisure time; $3=$ Environment, nature and animal welfare; $4=$ Churchly or religious sector; $5=$ Social and health sector; $6=$ Policy work and representation of interests; 7=Civic and local community activities; 8=Education; 9=Sports and exercise; 10=Neighborhood support and informal sector

\section{Monetary Valuation of Voluntary Engagement}

This chapter uncovers additional insights about the monetary influence of voluntary engagement. We extend this line of research by quantifying the total costs of voluntary contributions in the recent discussion on documenting the amount of performance of volunteers in monetary relationships. Our work employs the use of the average gross annual income including a gender specific perspective due to wage tax data stemming from 2010 considering full-time and all year permanence of employment. This points to a specific role of employers' expenses as vehicles of annual compensation schemes. Hence, limits of the monetary valuation of voluntary engagements arise, since the characteristics of individual contributions may give rise to theoretically misinterpreting certain activities due to prevailing classification asymmetries of the ten sectors of voluntary engagement.

Our analysis relates the annual total employers' expenses derived from wage tax data supplied by STATISTICS AUSTRIA to the timely efforts made by individuals assuming that these persons would supply a workload of 33.6 hours per week by multiplying these values resulting in the total costs of voluntary contributions in annual terms. Prior to the communication of the value of 33.6 effective working hours we calculated that voluntary engage ment in terms of permanent employment corresponds to 99,743 employees relating to supplied figures by STATISTICS AUSTRIA (2011) and its labor market report from the second quarter of 2011. These somewhat intuitive results possess an uncertainty component from a statistical point of view, since they carry the remedy of multiple assumptions, because of the reported nature and extent of involvement. We document, however the total costs of voluntary contributions amounting to $€ 3,298,128,405$.

Figure 3: Gender-specific total costs of voluntary contributions in million $€$ in fields of voluntary engagement 


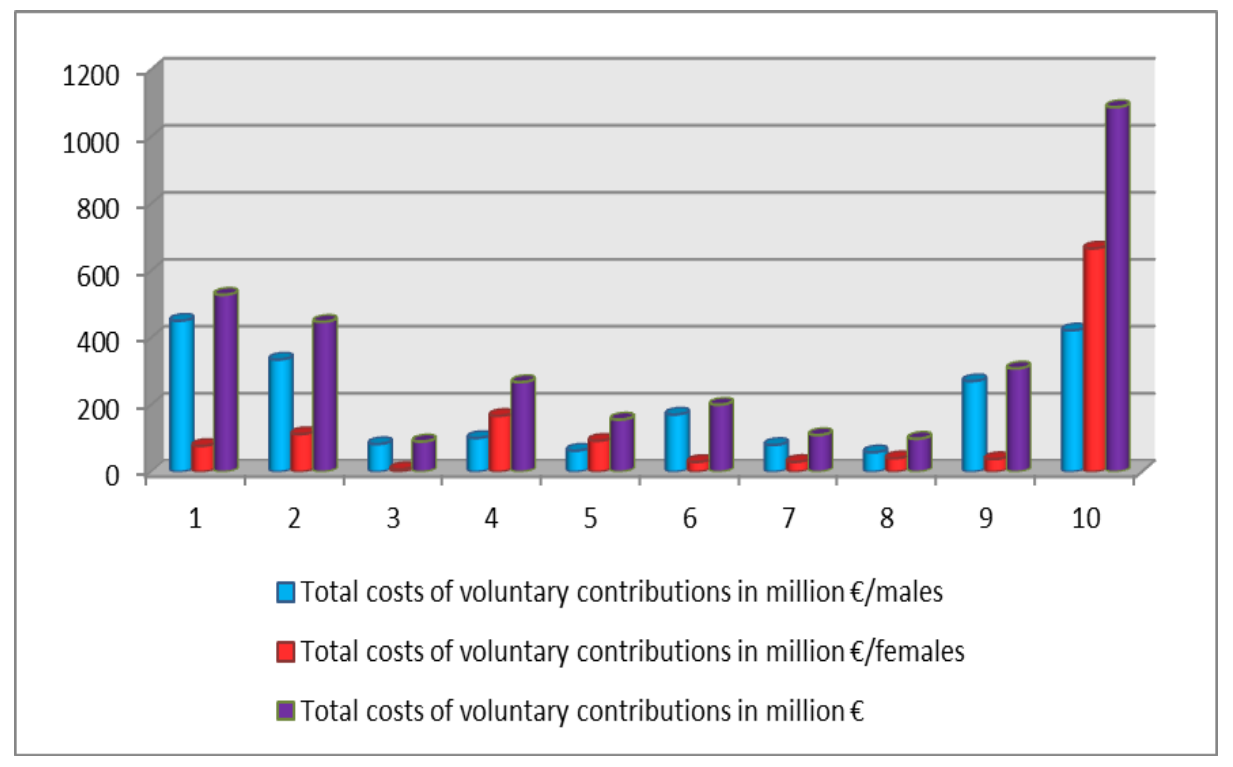

Source: STATISTICS AUSTRIA Wage Tax data 2010 and authors'own calculations.

Horizontal notations: 1=Emergency relief and rescue service; 2=Arts, culture, entertainment and leisure time; 3=Environment, nature and animal welfare; 4=Churchly or religious sector; 5=Social and health sector; 6=Policy work and representation of interests; 7=Civic and local community activities; 8=Education; 9=Sports and exercise; 10=Neighborhood support and informal sector

Figure 3 above demonstrates the gender-specific total costs of voluntary contributions in all ten fields of voluntary engagement. We find two directions of deviations in terms of gender-specific total costs of voluntary contributions. The first direction which prevails in the areas of formal voluntary engagement points to females total costs being significantly lower than of males: the most striking difference is in the area of "Environment, nature and animal welfare" where women's total costs marginally resemble $9.44 \%$ of the total costs of males and second ranked in the area of "Sports and exercise" where women's total costs amount to a mere $14.05 \%$ of the total costs of males in terms of employment data on a full time scale. The second direction aims in the opposite direction: in the field of "Neighborhood support and informal sector" the total costs of voluntary contributions of females exceeds the costs of males by $58.0 \%$ and in the formal field of "Churchly or religious sector" the total costs of females even exceed the costs of males by an impressive $65.5 \%$.

Our analysis bears strong resemblance with the prevailing attitude of individuals and sadly of societies, where voluntary engagement does lack respect in particular with a gender-sensitive focus frequently underestimating the efforts undertaken by females, who are double burdened in terms of being on leave due to their children and in terms of being victims of unfavorable compensation schemes due to lower salaries on average compared to men's salaries and due to part-time work engagements.

\section{Voluntary Contributions in a Societal Context}

This paper extends the line of research on the motivations behind the traditional scope of voluntary contributions in a societal context suggesting that the main driver of voluntary engagement embeds itself via intrinsic motivation which at its core lies in parental initiation. Since we examine the monetary valuation of voluntary contributions in Lower Austria, we find a rich pattern of relations that cannot be explained by purely intrinsic motivational developments. Therefore, we acquire clearer insights into the societal and correspondingly into the monetary value of voluntary contributions by individuals as if they were emplo yees of organizations, institutions and associations.

The expert interviews contribute to a clear understanding of the monetary valuation of voluntary engagement, since they provide a stable and transparent picture of voluntary activities that would be absent and subsequently would eradicate certain fields of voluntary engagement or partially result in quality deterioration of emergency relief and rescue services (for instance fire safety) and of neighborhood support. The experts suggest relocating certain services and accounting for public funding and consequent as sociational responsibility of volunteering efforts.

We emphasize the notion that quantitative data certainly lacks adequate monitoring of voluntary engagements as a whole and that it can be complimented with examining the societal context and its primary point of concern: the management of volunteers throughout any region and throughout all fields of study has to be embedded in an organization which faces undoubtedly multifaceted challenges with respect to hiring volunteers, to accompanying and to coordinating these individuals, to continuously foster personal contact and most importantly to respect and to motivate persons who are prone to conflict situations and to psychological challenges.

In general, the majority of interviewed experts are in favor of voluntary contributions although they show disapproval towards monetary compensation of voluntary efforts and towards substituting volunteers by permanently employed individuals. Voluntary contributions may give rise to a pervasive and a positive multi-dimensional life satisfactory influence with the 
monetary compensation occupying solely an undervalued role in the societal context and in an intrinsic consideration. The concluding remark we point out with respect to the expert interviews on monetarily compensating voluntary engagement is that societal progress coupled with continuous individualization empowers persons to disentangle their social networks and to contribute to substantially merge different social groups and to interact not only on a regional level.

Public authorities have to provide an appropriate general legal framework and additionally the subject of insurance coverage for volunteers has to be more present not only in Austria, but in all EU countries. They should improve the collection of statistical information on volunteering as a support for the nations themselves as well as for the organizations. The cooperation between government and volunteer organizations is crucial for promoting volunteering activities.

\section{Summary}

This paper studies voluntary contributions in Lower Austria in specific terms by matching the monetary value of voluntary contributions in order to gain better insight into the monetary valuation of voluntary contribu tions which has largely remained unexamined due to the vague and challenging description of their content. In general assumptions, voluntary engagement is don e voluntarily, without payment, outside one person's own household for external purposes, and embedded into an organization, into an as sociation, into an institution or in form of neighborhood support without any institutional boundaries.

This voluntary engagement absent of any monetary compensation is the main point of identification of this paper be ing achieved through quantitative and qualitative analysis. We depart by tracking a cumulative data file from STATISTICS AUSTRIA in terms which purpose is twofold: first, in order to estimate the quantitative dimension of voluntary contributions, data from an additional section of the Austrian Micro Census is considered, and second employment and unemployment data from the section of the Austrian Micro Census that covers the European Labor Force Survey, to provide monetary valuation of voluntary contributions. Expert interviews are employed to round up the qualitative analysis of voluntary engagement.

We find that in the year of 2006 a total of 617,804 persons in Lower Austria - which amounts to 46.8\% of the population - are voluntarily engaged in the field of neighborhood support as well as in different organizations, institutions and associations. The crucial point we wish to make here is that this impressive number of volunteers refers to persons who are above 15 years of a ge and who primarily reside in Lower Austria. Our result suggests that the Austrian civil society mainly in rural areas is frequently constrained due to a weak identification scheme with the civil society.

In terms of gender composition we show that 321,669 men versus 296,139 women are voluntarily engaged. In the formal field of voluntary involvement male engagement prevails, whereas in the informal field the amount of female volunteers prevails, due to the fact that $67.5 \%$ of all women above 15 years of age are present in the field of neighborhood support. The quantitative dimension of voluntary contributions is also at its maximum in the field of neighborhood support in Lower Austria with a weekly amount of 886,434 hours in total. As far as the formal field of voluntary involvement is con sidered, the highest rank is attributed to the "Churchly or religious sector" with 118,295 persons, followed by the area of "Emergency relief and rescue service" with 103,598 persons and by the sector of "Arts, culture, entertainment and leisure time" with 100,298 volunteers.The collective challenges for volunteering in Europe, on the national as well as on the European level, are identified as the matching of the needs of volunteers and organizations, the difficulty for organizations to find people that are willing to volunteer for the long term and/or are ready to assume responsibility in organizations and the interaction between volunteers and full-time staff serious professionalization vs. integration of volunteers (Annual Report European Volunteer Cen tre, 2012).

Our paper was partially motivated by shedding a light on the interdependences between the quantitative and the qualitative analysis of the broad scope and of the limitations of voluntary engagement. The objective of this work was to demonstrate that the sheer focus on the monetary valuation of voluntary contributions of 617,804 Lower Austrians needs to be extended by considering not only its societal benefits but its purpose for the volunteers themselves as far as their social environment is taken into account.

\section{References}

Angermann, A. and Sittermann, B. (2010) Volunteering in the European Union - An Overview. Working paper no. 2 of the Observatory for Sociopolitical Developments in Europe.

Annual Report European Volunteer Centre (CEV) - 2011 (2012) accessed on 08-22-2012 at:

http://www.cev.be/wp-content/uploads/2012/06/CEV_Annual_Report_2011.pdf

European Commission (2007) European Social Reality. Special Eurobarometer 273 accessed on 10-10-2012 at:

http://ec.europa.eu/public opinion/archives/ebs/ebs 273 en.pdf

Österreichischer Rat für Freiwilligenarbeit (2004) Aktionsprogramm Freiwilligenarbeit. Bundesministerium für soziale Sicherheit, Generationen und Konsumentenschutz: Wien.

STATISTICS AUSTRIA (2006) Mikrozensusdaten 4. Quartal 2006 inklusive Freiwilligenarbeit, Wien.

STATISTICS AUSTRIA (2011) Mikrozensus Arbeitskräfteerhebung 2. Quartal 2011, Wien.

Wollebæk, D., Skirstad, B. and Hanstad, D.V. (2012) Between two volunteer cultures: Social composition and motivation among volunteers at the 2010 test event for the FIS Nordic World Ski Championships. International Review for the Sociology of Sport, doi:10.1177/1012690212453355 